\title{
Some bacteria more than sum of parts
}

\author{
Gut bacterial aggregates as living gels \\ Authors: Brandon H Schlomann, and Raghuveer Parthasarathy \\ https://doi.org/10.7554/eLife.71105 \\ Recommended with a Commentary by Srividya Iyer-Biswas, \\ Purdue University and Santa Fe Institute
}

A popular science talk by the inimitable Bonnie Bassler challenges our naive notions of organismal identity: she points out that we are mostly bacteria - for every "human" cell, our bodies have $\sim 10$ bacterial cells, and for every "human" gene, around $\sim 100$ bacterial genes! An important assembly of bacteria in animals, including humans, is the gut microbiome, an extraordinarily complex ecosystem composed of a wide variety of microbes living together in a crowded environment. Moving beyond scrutiny of species composition within this ecosystem, one can ask if/how the physical organization of bacteria within the gut microbiome influences population growth as well as host health. A major challenge in addressing this question has been that quantitative characterization of this spatial structure requires three-dimensional data gathered from living hosts, a feat that long proved experimentally intractable. Recent advances in microscopy have tipped the balance, however, enabling researchers to obtain high-precision dynamical data of gut microbiomes for the first time. In this publication, the authors construct a quantitative framework to describe the observed distribution of bacterial cluster sizes within the gut microbiome and show that this system behaves like a "living gel".

Light-sheet fluorescence microscopy uses a plane of light to optically section a specimen, allowing the reconstruction of three-dimensional images of tissues with subcellular resolution. The authors have taken advantage of this approach to gather three-dimensional images of the complete intestines of live zebrafish larvae with defined microbiota. Zebrafish larvae have the attractive feature that they are optically transparent. In addition, they are anatomically similar to other vertebrates, including humans, and amenable to manipulations that allow the study of one gut bacterial species at a time. To image the entire volume of the gut, corresponding to $\sim 1.2 \mathrm{~mm}$ in length, the authors leveraged the speed of coverage facilitated by light-sheet based imaging. Naturally, accurate measurements of cluster sizes require rapid and accurate imaging of the entire volume of the gut, as slower measurements of such a large volume risk introduction of artifacts due to convection and movement of smaller clusters into the imaging volume. An added advantage of this method is that the light sheet is remarkably photo gentle, owing to excitation being restricted to the image plane being captured. This preserves the fluorescence across the volume of the sample vis-à-vis other imaging modalities, while also providing excellent contrast.

For this study, each zebrafish gut microbiome contained only one of eight different fluorescently labeled bacterial strains, with each strain exhibiting a variety of behaviors and 
population dynamics. The authors first observed that, in all cases, the bacteria form clusters, ranging in size from individual cells up to conglomerates that fill almost the entire gut. The statistical distribution of object sizes is an important feature that has been used to understand the underlying generative mechanisms in a plethora of systems, ranging from sizes of galaxy clusters to gel formation in polymer solutions. For this reason, the authors first calculated the reverse cumulative distribution of cluster sizes for each strain, which describes the probability that an intestinal aggregate will contain more than a certain number of bacterial cells. Despite substantial variation across individual fish, the trends for each strain exhibited the same general features: (i) a power-law-like decay for small cluster sizes, with a similar exponent for all strains, which (ii) becomes shallower for larger cluster sizes in a strain-dependent manner. That is, the similarity in the signatures of spatial organization is captured by a specific mathematical form for the likelihood that a given bacterial cluster is of a particular size.

These shared features suggested the influence of generic (and not strain-specific) processes, which the authors next sought to model by considering the four most relevant biophysical processes: growth due to division of single cells, fragmentation due to escape of single cells from clusters, aggregation due to merger of distinct clusters, and expulsion due to removal of clusters by transit out of the intestine. Clusters grow through cell division, resulting in their size growing at an exponential rate (growth rate). Clusters can decrease in size through fragmentation as single bacteria escape from them. The fragmentation rate is formulated to encompass, through parametric variation, a broad range of mechanisms including cases in which (i) all cells are equally likely to fragment; (ii) only cells at the surface of the cluster fragment; and (iii) all clusters have the same fragmentation rate irrespective of size, mimicking a chain of cells where only the cells at the ends of the chain can break off. The authors incorporated the limited capacity of the gut into the model by decreasing the growth and fragmentation rates as the total number of cells approach the carrying capacity. Aggregation is the process through which two clusters join together during intestinal mixing to form a single cluster, the size of which is equal to the sum of sizes of the two individual clusters. The rate of aggregation is determined by the product of sizes of the two individual clusters. Finally, expulsion is the process through which clusters are removed from the system by transiting along and out of the intestine. The expulsion rate of clusters of a given size is itself determined by the cluster size.

The authors found that growth and fragmentation are especially important in determining the distribution of small clusters, irrespective of species. They describe these processes in a manner reminiscent of the roles of reproduction and mutation in determining the population distributions that arise in the context of evolutionary genetics. (For stochastic processes aficionados, there is an analogy to the Yule-Simons process, a classic populations genetics model describing the distribution of allele frequencies, which follows the same power law.) Modifying this process to account for the influence of spatial structure and the finite carrying capacity of the gut results in a distribution that accurately accounts for the experimental data, with both a power-law-like behavior at smaller cluster sizes as well as a plateau at larger cluster sizes. Interestingly, this overall process resembles the gelation transition in soft materials, leading the researchers to label the gut microbiome as a living gel. Is this an evocative pictorial analogy, or there is something deeper there? Remains to be seen.

This model successfully captures the experimentally observed size distribution of bacterial 
clusters and also clearly distinguishes the individual effects of each process, while adding in complexity layer by layer. The generality of this model hints at a broad applicability to other organisms, offers cluster size distribution as a potential biomarker to study health issues related to the microbiome, and suggests a number of follow-on experiments to further quantify the physical and biological processes responsible for shaping the spatial structure of the gut microbiome. These findings open the possibility of inferring information about gut microbiota in organisms which are not amenable to direct imaging, such as humans. The responses of bacterial colonies in the human gut to stimuli like drugs or pathogenic invasions cannot be observed directly. However, snapshots of bacterial clusters could be gathered from, for example, fecal samples. The findings in this work suggest that there may be a path from the latter to the former - in other words, the ability to examine static information of microbiota spatial structures and infer the biological dynamics that created them.

In sum, this work showcases the success that can be had in investigations of complex and functional living systems through a synthesis of high-precision dynamical measurements and first-principles-based theory. Using iterative feedback between experiment design and theory frameworks, the authors have identified emergent simplicities in the spatial arrangement of gut bacteria and related them to the biophysical processes implicated in the formation of such spatial arrangements in the gut. The skeptic may argue that "power law blah blah blah" seldom leads to mechanistic insights of biological significance - while simple and elegant, is the model functionally relevant? Follow-up investigations could potentially explicate the mechanisms by which colony structure forms, how the structure relates to the activity of gut microbes, and how the structures may be altered during perturbations such as invasions by new species or antibiotic treatments.

Acknowledgements: We thank Kunaal Joshi, Charlie Wright, Senthil Arumugam, Sean Gibbons and Raghuveer Parthasarthy for useful discussions. 